\title{
Plug-and-Play Distributed Algorithms for Optimized Power Generation in a Microgrid
}

\author{
Emanuele Crisostomi, Member, IEEE, Mingming Liu, Student Member, IEEE, Marco Raugi, and \\ Robert Shorten, Senior Member, IEEE
}

\begin{abstract}
This paper introduces distributed algorithms that share the power generation task in an optimized fashion among the several Distributed Energy Resources (DERs) within a microgrid. We borrow certain concepts from communication network theory, namely Additive-Increase-Multiplicative-Decrease (AIMD) algorithms, which are known to be convenient in terms of communication requirements and network efficiency. We adapt the synchronized version of AIMD to minimize a cost utility function of interest in the framework of smart grids. We then implement the AIMD utility optimisation strategies in a realistic power network simulation in Matlab-OpenDSS environment, and we show that the performance is very close to the full-communication centralized case.
\end{abstract}

Index Terms-Distributed algorithms, microgrid, optimal power scheduling.

\section{INTRODUCTION}

A N objective of the smart grid is to perfectly balance supply and demand in the power network [1], [2] and [3], and achieving such an objective with a high level of reliability when a large share of power is generated from renewable fluctuating resources is one of the current challenges in the power community. The main challenges in realizing this aspiration arise because the power network is very large scale, and each node of the network should sense in real-time its required energy demand, and then try to balance this demand with the amount of produced energy [4]. Clearly, not only the generated power from renewable sources is stochastic, but the demand is also uncertain as it depends on the end-users usage of electric appliances. Energy usage is also further affected by highly unpredictable factors, such as energy price, weather conditions (use of air conditioning/ heating), and (in the near future) also by an increasing penetration of electric transportation [5]. Thus, due to the large number of distributed energy resources (DERs) and (aggregates of) endusers, the real-time power scheduling and balancing problem is currently an important and challenging area of research.

Virtual Power Plants (VPPs) are currently seen as a viable answer to the increasingly distributed nature of the power system network [6]. A Virtual Power Plant is defined as "a cluster of

Manuscript received April 18, 2013; revised December 10, 2013, March 21, 2014; accepted April 21, 2014. Date of current version June 18, 2014. This work was in part supported by Science Foundation Ireland under grant 11/PI/1177. Paper no. TSG-00303-2013.

E. Crisostomi and M. Raugi are with the Department of Energy, Systems, Territory and Constructions Engineering, University of Pisa, Pisa 56126, Italy.

M. Liu is with the Hamilton Institute, National University of Ireland, Maynooth, Ireland.

R. Shorten is with IBM Research Ireland, Dublin 4, Ireland.

Digital Object Identifier 10.1109/TSG.2014.2320555 dispersed generator units, controllable loads and storage systems, aggregated in order to operate as a unique power plant" [7]. The term virtual refers to the fact that the VPP will be, in general, a multi-fuel, multi-location, and multi-owned power station. From a grid operators perspective, purchasing energy or ancillary services from a virtual power plant is equivalent to purchasing from a conventional station [2]. The idea of a VPP is illustrated in Fig. 1. In practice VPPs decompose the original complex fully centralized network into a number of distributed units, each one of which has a central entity, called an Energy Management System (EMS), that performs the balancing task. Every time balancing is not achieved (e.g., the power generated within a VPP, plus that stored in available storage systems is not enough to fulfil the end-users requirements), then the VPP buys energy from other VPPs, or from the smart grid in general, or can curtail some controllable loads (e.g., Direct Load Control, see [8]). Similarly, if more energy than required is produced, then the VPP tries to sell energy to other VPPs.

The microgrid shares similar features with the VPP, but usually the term VPP is used to emphasize the economic features of the structure, i.e., the ability to compete in the energy markets against other VPPs. On the other hand, the microgrid must be able to operate in a grid-connected mode, isolated mode, and in a transition between grid-connected and isolated modes [9]. One of the main advantages of microgrids and VPPs is that the energy demand will be primarily satisfied by the DERs belonging to the same cluster of the users, thus generally avoiding long distance transport of energy and satisfying the small-distance producer-consumer paradigm [3].

The main task of the EMS of a microgrid is to achieve the aforementioned balance of produced/consumed energy. Although the size of a microgrid is much smaller than that of the whole smart grid, there is still the major problem of selecting which power generators to use, and in which proportion, to provide the required power.

Remark: Note that the DERs within the same VPP or microgrid do not compete among themselves to increase their earnings, as overall they constitute a single unique (virtual) power plant. In fact, the DERs cooperate to achieve a common goal, e.g., minimize the sum of the financial costs incurred by each DER to generate its share of power.

\section{A. Paper Contribution}

The main contribution of this paper is to design an algorithm that automatically shares the power generation task among the available DERs in a way that is fair and distributed. As we shall see, the concept of fairness in our context will be with respect 


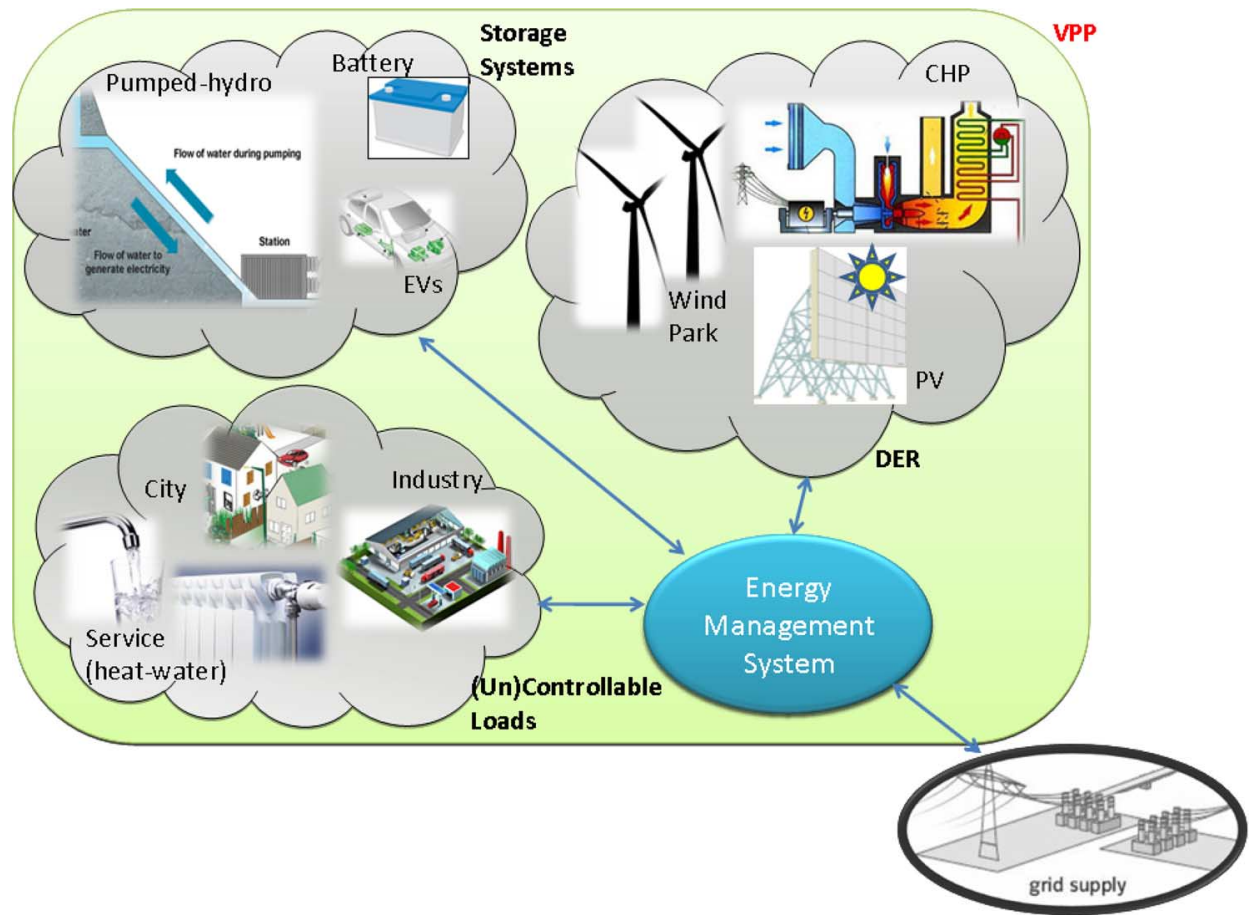

Fig. 1. A Virtual Power Plant (VPP) is generally characterized by Distributed Energy Resources (DERs), including Combined Heat and Power (CHP) and wind/ solar plants, storage systems, controllable and uncontrollable loads, Electric Vehicles (EVs), and is connected to other VPPs through the grid.

to a utility function, where utility will refer to financial costs. We will achieve this objective by adopting AIMD (Additive Increase Multiplicative Decrease) algorithms, which are borrowed from communication network theory [10]. In this context, our paper provides several contributions: (i) we propose a truly distributed solution to the power generation problem, which at the same time minimizes communication overhead; (ii) we present a consensus-like modification of the conventional synchronized AIMD algorithm to solve the utility minimization problem in the case of quadratic cost functions; and (iii) we illustrate our results in a realistic simulation in a Matlab/OpenDSS framework.

This paper is organized as follows. The next section reviews the state of the art for power generation in a smart grid context. Section III describes the power generation problem and the financial cost function of a microgrid. Section IV describes the basic AIMD algorithm, and how it can be adapted to the power generation context. In the same section we also explain how the algorithm can be modified to minimize the power generation cost in a distributed manner. Section V compares the performance of the proposed algorithms with that of a fully centralized algorithm, in a Matlab-OpenDSS environment. Finally in Section VI we summarize our findings and outline future lines of research.

\section{STATE OF THE ART}

Balancing the energy demand and the energy offer is a challenging problem due to a number of effects.

- The uncertain demand. This refers to the uncertain energy consumption from both industries and single users. Many authors have addressed this problem using techniques from machine learning and time series analysis in an attempt to accurately forecast the power load [11]-[13] and [5].

- The uncertain energy offer. As the penetration level of energy being produced from renewable sources is constantly increasing, the availability of energy is highly affected by weather conditions (i.e., availability of sun/wind). More accurate weather forecast services, together with an extensive use of storage systems, are currently used to mitigate such uncertainties [14].

A first approach to optimally share the power generation task among the DERs, is to let the EMS solve the optimal scheduling problem at fixed time steps, typically chosen between 5 and 30 minutes. This centralized solution has the main drawback of a significant communication overhead, as every single DER must communicate to the EMS how much power can be provided. Then, the EMS must gather all this information, and the energy needed by the users, solve an optimization problem, and communicate back to each DER its correct allocation of energy. See for instance [15], [16] and [17] for similar approaches for optimal power scheduling.

To avoid this frequent exchange of information, some authors have designed day-ahead thermal and electrical scheduling algorithms of large-scale VPPs (LSVPPs) [18], [19], or [20] where the day-ahead unit commitment problem is solved in a distributed fashion. In [21] the day-ahead solution is then corrected in a centralized framework, considering latest available measurements. In fact, in the afternoon prior to the scheduled energy delivery, the hourly prices for the following day (set by electricity spot markets) are already known, and at the same time, quite accurate weather and load forecasts are also available [18]. This approach is for the most part effective. However, it does not consider real-time information, such as the error between the available power and that predicted on the basis of weather forecast, that could be important to fully exploit the renewable resources. In addition, this solution is not robust, and problems in the EMS will have consequences in the entire network in terms of grid stability. 


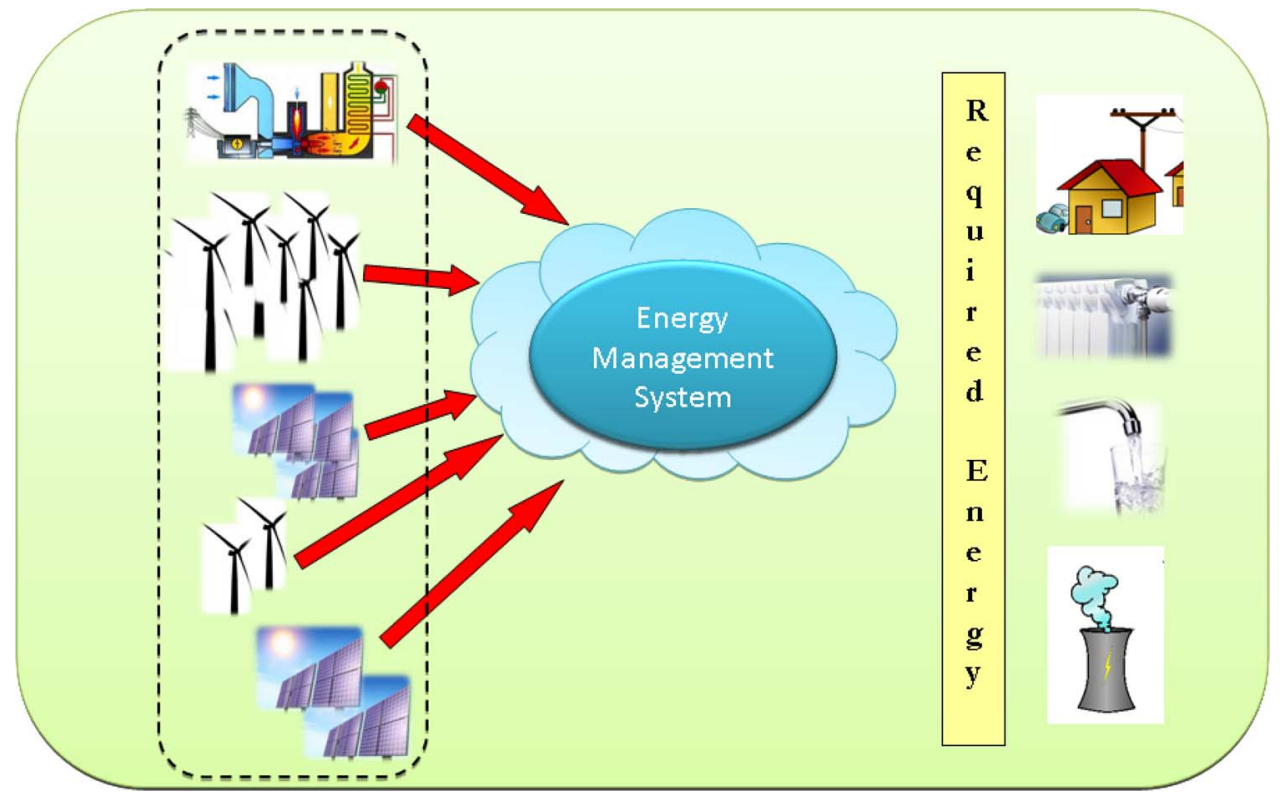

Fig. 2. DERs (on the left) must provide enough energy to satisfy the demand coming from the users (e.g., from industries and residential areas). AIMD algorithm is suggested to coordinate the DERs.

Recently, there has been a strong trend in many different engineering disciplines to move from centralized strategies to distributed ones. Examples of this trend can be found in Internet research, electric vehicle charging and indeed in the case of demand side management, see for instance [22]-[26] and [27]. Distributed systems do also provide the benefit that DERs can join and leave the power generation task at will without any centralized supervision, thus giving rise to a system that is "plugand-play" in spirit. This latter point allows entities with excess power generation (e.g., PVs in residential areas in summer days) to profit from underutilized resources. The main objective of this paper is to follow such a distributed trend. Specifically, to design an algorithm that automatically shares the power generation task among the available DERs in a way that is fair and distributed.

\section{Problem Statement and Preliminaries}

We consider the scenario depicted in Fig. 2, and assume that at a given time $t$, each one of $n$ DERs provides an amount of power denoted as $p_{i}(t)$, where the index $i$ refers to the $i$ 'th DER. Our main assumption is that at every time step $t$, the overall set of DERs is always able to provide the total power $d(t)$ required by the users. Therefore, the EMS has the ability to choose which of the DERs will be used, and in which proportion, to provide the requested power.

Remark: Note that the assumption that the power requested by the users is smaller or equal than that provided by the DERs is not a strong assumption. In fact, if this is not true, then the EMS can either take more energy from storage systems, or buy it from other VPPs or from the outer grid. It may also decide to disconnect some of the loads, thus reducing the demanded power. After completing any of the previous actions, the condition that the energy produced by the DERs is enough to satisfy the (residual) demand holds. Many papers in the literature have tackled the problem of which of these actions is more convenient from the EMS point of view; see for instance [15], [16] and [17] that propose optimal scheduling solutions. However, this problem is not of interest here. In this work it is more convenient to simply assume that the demand is smaller than the power deliverable from the DERs; otherwise, the load is provided in a best effort fashion.

As the DERs provide more or equal power than is required by the users, the purpose of this paper is to automate this process in a decentralized manner such that some utility function of interest is minimized; namely, the financial cost associated with power generation.

\section{A. Utility Minimization}

In principle, the EMS has many ways of sharing the power demand among the available DERs. We are interested in the power share that minimizes the financial cost of producing the desired power. To solve this problem, we associate each DER with a quadratic utility function, as common in the VPP literature, see [28] and [16].

$$
C\left(P_{g}\right)=a \cdot P_{g}^{2}+b \cdot P_{g}+c
$$

where $C$ is the hourly cost in Currency Unit (C.U.) per hour, $P_{g}$ is the generated power in $\mathrm{MW}$, and $a, b$ and $c$ are coefficients of appropriate measurement unit that depend on the technology of the power plant (e.g., fuel cost, efficiency, etc.). In particular, $b$ includes operation and maintenance costs (O\&M) costs, and fuel and carbon costs, which are usually expressed in $\mathrm{E} / \mathrm{MWh}$ (or in $\$ / \mathrm{MWh}$ ). The coefficient $c$ takes into account the expenses that are incurred even if no energy is produced at all. Quadratic cost functions have been used in many references in the literature, see for instance the classic [29], or the more recent [28] and [16] in the context of VPPs. Due to the fact that the coefficients $a$ are usually quite small, and because of the difficulty of handling nonlinear (though quadratic) cost functions, many 
authors in the literature simply neglect the quadratic term, and use affine functions in the optimization; see for instance [15]. Also note that in the literature it is very simple to find databases containing values of coefficients $b$ and $c$ for several examples of DERs all over the world, for the computation of the levelized costs of electricity (LCOE) [30].

In this paper we use the complete quadratic cost function (1). However, the study can be extended to other convex utility functions of interest ${ }^{1}$; for instance, one could minimize an environmentally friendly utility function (e.g., $\mathrm{CO}_{2}$ emissions) to incentivate the penetration of renewables.

\section{AlgorithMS FOR UTILITY MiNIMIZATION FOR VPPS VIA SYNCHRONIZED AIMD}

The problems that we described are very close to those encountered in Internet congestion control. In Internet congestion control, one tries to allocate bandwidth such that certain objectives are realized [31]. A wide variety of work has been published in this area and it can be shown that the TCP Reno and TCP Vegas both correspond to various utility maximization solutions [31]. In AIMD an individual agent (e.g., a computer sending packets) gently increases its transmission rate, during the Additive Increase (AI) phase, until a packet loss signal is received. This is called a congestion event, and indicates that the sum of individual bandwidths has exceeded the total capacity. Upon detecting congestion, the agents instantaneously decrease their transmission rate in a multiplicative fashion. This is the Multiplicative Decrease (MD) phase of the algorithm [10]. Note that such congestion control problem in the Internet exhibits similar characteristics as power generation here. The quantities of interest are positive (bandwidth/power), locally bounded (local maximum transmission rate/available power), the available capacity/required energy may vary over time, and the system of interconnected agents (Internet/Smart Grid) is very large scale. We refer the reader to [10], [32]-[35] for a discussion on convergence properties of the AIMD algorithms.

\section{A. AIMD}

In this section we borrow ideas from the Internet congestion control community, and we adapt the basic TCP to the scenario of interest here, as depicted in Fig. 2.

\section{Algorithm IV.1: BASIC AIMD Algorithm $\left(p_{i}\right)$}

$$
p_{i}(0)=p_{i 0}
$$

\section{repeat}

$$
\begin{aligned}
& t=t+1 \\
& \text { if } \sum_{i=1}^{n} p_{i}(t)<d(t) \\
& p_{i}(t+1)=\min \left[p_{i}(t)+\alpha_{i}, \bar{p}_{i}(t)\right], \forall i=1, \ldots, n
\end{aligned}
$$

\section{else}

$$
p_{i}(t+1)=\max \left[\beta_{i} p_{i}(t), \underline{p}_{i}(t)\right], \forall i=1, \ldots, n
$$

until end of simulation

\footnotetext{
${ }^{1}$ This is the subject of current work.
}

In Algorithm IV.1, the parameter $\alpha_{i}$ is the positive additive parameter associated with the additive increase phase of the algorithm (AI) (expressed in $\mathrm{kW}$ or $\mathrm{MW}$ ), and $0<\beta_{i} \leq 1$ is the multiplicative parameter used in the decrease phase (MD). The quantities $p_{i}(t)$ and $\bar{p}_{i}(t)$ denote the minimum and maximum bound on the power respectively. In fact, the values of $p_{i}$ cannot take arbitrary values due to: (i) the limited sizes of the DERs; (ii) the availability of renewables (sun/wind); (iii) the power network constraints (e.g., transformer tap settings, security constraints, minimum voltages at load buses and transmission lines); and (iv) due to the fact that DERs can modulate their power generation without exceeding a nominal ramp reference. Note also that such bounds are time varying.

Remark: The main feature that makes the AIMD algorithm particularly convenient to apply in large-scale systems, is that the algorithm can be easily implemented in a truly distributed manner. In fact, Algorithm IV.1 only requires the EMS to send a "congestion notification" to the DERs when the produced power equals the demanded power, which can be coded in a single bit of information. In the context of power balancing, such a notification will be denoted as balancing notification in the remainder of the paper. This implies that the DERs do not have to exchange information among themselves, do not have to communicate the available sun/wind to the EMS, and also do not even have to communicate when they stop or start contributing to the power generation task. Furthermore, the EMS itself does not have to communicate to the DERs how much power they must provide.

In order to illustrate in a simple fashion the mechanism of the basic AIMD Algorithm IV.1, Fig. 3 illustrates the output of the algorithm in a simple toy example, which corresponds to the VPP scenario investigated in [15]. Therefore, we assume that three DERs are available: a PV plant; a wind plant; and a CHP. It is assumed that the PV plant has a net capacity of $6 \mathrm{MW}$ and a capacity factor of $6 \%$; the wind plant has a net capacity of $45 \mathrm{MW}$ and a capacity factor of $27 \%$; the CHP plant has a net electrical capacity of $40 \mathrm{MW}$ and a capacity factor of $85 \%$. We assume that all DERs have the same $\alpha_{i}$ and $\beta_{i}$ parameters equal to $0.01 \mathrm{MW}$ and 0.95 respectively. Fig. 3(a) illustrates the case without constraints (i.e., without even considering the sizes of the power plants), when the power demand is constant and equal to $35 \mathrm{MW}$. Note that in this case AIMD gives rise to the fair solution where each single DER produces exactly the same quantity of energy. The smaller sizes of the renewable energy sources, together with renewable availability (e.g., no sun at night time), make the AIMD solution provide completely different results in Fig. 3(b) than those depicted in Fig. 3(a). The case study scenario is then further complicated, assuming a more realistic non-constant power demand, and the corresponding results are shown in Fig. 3(c) (the time-varying demanded power profile is taken again from [15]). In the same scenario, Fig. 3(d) compares the power demand with the power generation of the example depicted in Fig. 3(c). Note that the individual power generated by the three single DERs are now summed, and, as expected, demand and offer are perfectly balanced.

Remark: The algorithm illustrated in IV.1 gives rise to sawtooth signals. Note that not all equipment might be suitable to handle such signals. Thus, typical methods that are already used to handle oscillating power generated from renewable sources 


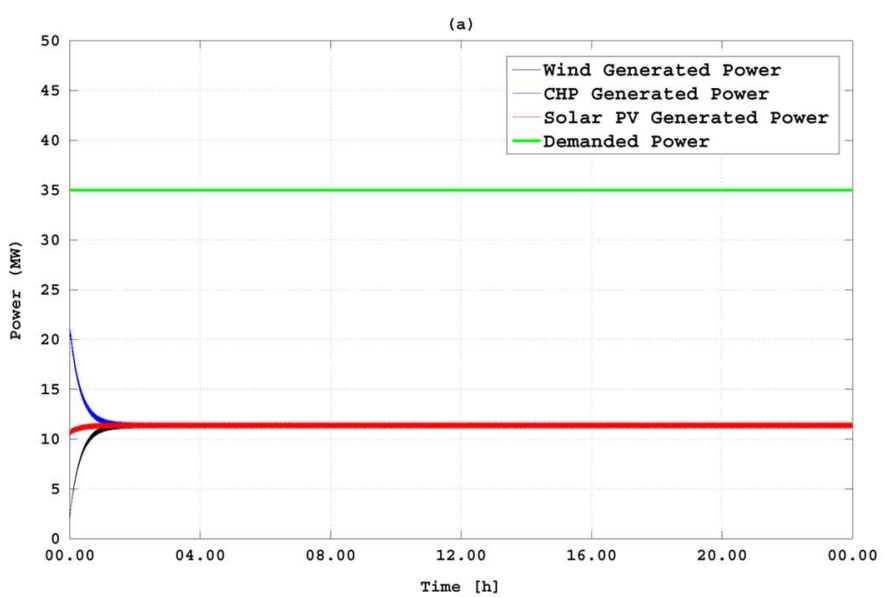

(c)

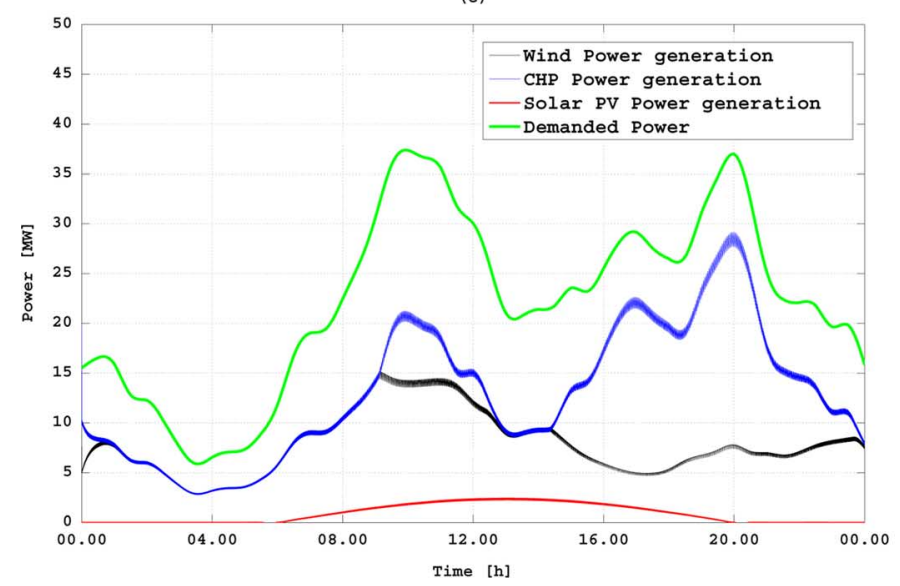

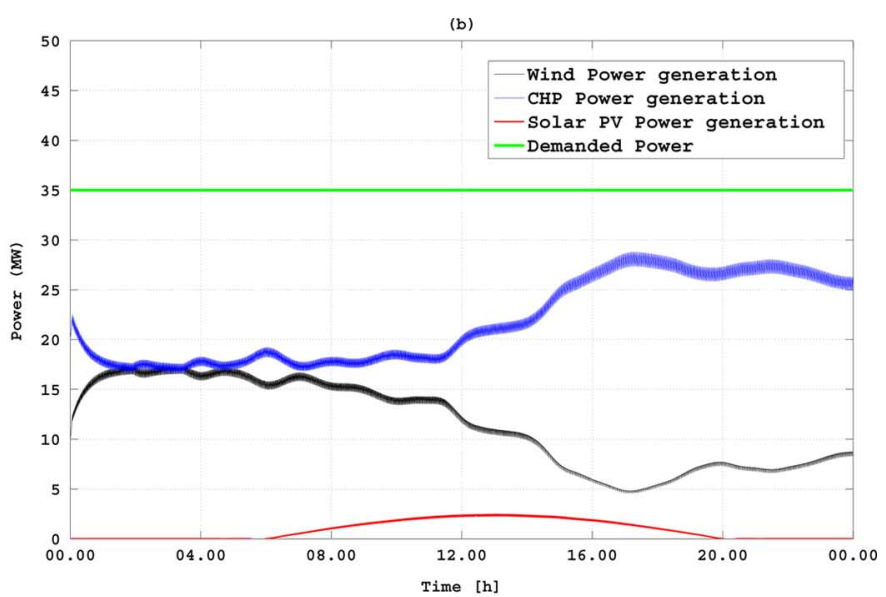

(d)

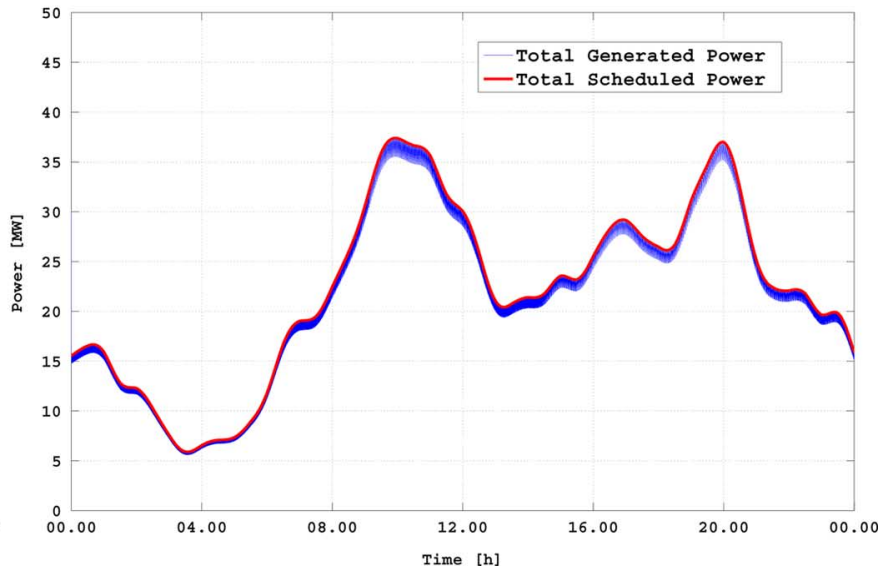

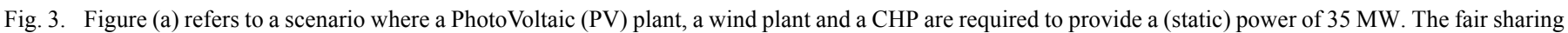

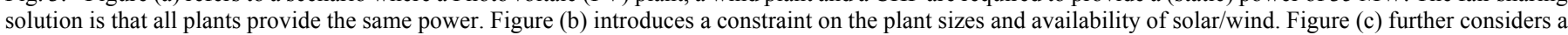
typical non-constant requirement of energy, and Figure (d) shows the required and provided power (summing contributions of single plants) of scenario (c).

might be required (e.g., PI-smoothing of the output power, prefiltering of the AIMD signal, use of batteries for primary frequency and voltage control).

\section{B. Utility Optimization via Synchronized AIMD}

The basic AIMD algorithm is designed to share the required energy among the available DERs in some manner. For instance, if there were no constraints, then each DER would provide the same quantity of energy as illustrated in Fig. 3(a). In this section we show that by appropriately modifying the basic AIMD algorithm, it is possible to minimize the financial cost of generating the required power, according to the cost functions introduced in Section III.A. Similar work in this direction can be found in [36]. The optimization problem can be formally stated as

$$
\begin{aligned}
& \min \sum_{i=1}^{n} f_{i}\left(p_{i}(t)\right) \\
& \text { s.t. } \sum_{i=1}^{n} p_{i}(t)=d(t)
\end{aligned}
$$

i.e., at every time step $t$ we want to find the combination of $p_{i}(t)$ such that the generated power is equal to the requested power, and it minimizes the sum of the cost functions. Each utility function has the form $f_{i}\left(p_{i}(t)\right)=a_{i} \cdot p_{i}(t)^{2}+b_{i} \cdot p_{i}(t)+c_{i}$, as was described in Section III.A. Note that in this section we do not consider the constraints on the single $p_{i}(t)$, as the AIMD algorithm will automatically satisfy them as described in Section IV.A. This minimization problem can then be conveniently solved at each time step $t$ with the aid of Lagrange multipliers, and the solution is known to be

$$
p_{i}^{*}(t)=\frac{\lambda^{*}(t)-b_{i}}{2 a_{i}}
$$

where

$$
\lambda^{*}(t)=\frac{d(t)+\sum_{i=1}^{n} \frac{b_{i}}{2 a_{i}}}{\sum_{i=1}^{n} \frac{1}{2 a_{i}}}
$$

subject to the linear constraint being satisfied, and where the uniqueness of the solution follows from the convexity of the utility functions ${ }^{2}$. Our approach is similar, but we solve the minimization problem by achieving consensus on the value of the

${ }^{2}$ The KKT conditions are necessary for optimality. By placing extra conditions on the utility functions they are also sufficient. 
function $\lambda_{i}(t)=\partial f_{i}\left(p_{i}(t)\right) / \partial p_{i}(t)[37]^{3}$. For this purpose, the basic algorithm can be adapted for utility optimization purposes as follows:

\section{Algorithm IV.2: AIMD UTILITY OPTIMIZATION $\left(\boldsymbol{\lambda}_{\boldsymbol{i}}\right)$}

$$
\begin{aligned}
& p_{i}(0)=p_{i 0} \\
& \text { repeat } \\
& t=t+1 \\
& \text { if } \sum_{i=1}^{n} p_{i}(t)<d(t) \\
& \lambda_{i}(t+1)=\lambda_{i}(t)+\alpha_{\lambda}, \forall i=1, \ldots, n
\end{aligned}
$$

else

$$
\lambda_{i}(t+1)=\beta_{\lambda} \cdot \lambda_{i}(t), \forall i=1, \ldots, n
$$

until end of simulation

and the multiplicative step is performed when the sum of delivered power exceeds the requested power. All DERs have the same parameters $\alpha_{\lambda}$ and $\beta_{\lambda}$, so called to remind that they refer to an AIMD algorithm on the variable $\lambda$. Therefore, AIMD makes all $\lambda_{i}$ converge to a unique value which has to be $\lambda^{*}$. Note that each DER has to update its own variable $\lambda_{i}$, and the communication requirement is again only restricted to the balancing notification from the EMS.

\section{Practical Implementation}

The utility optimization problem can be theoretically solved by simply running an AIMD consensus problem on $\lambda_{i}$, as when all DERs have the same value of $\lambda_{i}$, then this value has to be $\lambda^{*}$. However, $\lambda_{i}(t)=\partial f_{i}\left(p_{i}(t)\right) / \partial p_{i}(t)$ is only an abstract quantity which, for the choice of utility functions in here, is $2 a_{i} p_{i}(t)+b_{i}$; it is not straightforward to compute how a DER can change its own value of $\lambda_{i}(t)$ by adjusting its power generation, and also how to express the power constraints in terms of $\lambda_{i}(t)$. Therefore, we now remap the AIMD algorithm in terms of $p_{i}(t)$ instead of $\lambda_{i}(t)$. By simply exploiting the fact that $\lambda_{i}(t)=2 a_{i} p_{i}(t)+b_{i}$, we can rewrite the AIMD Algorithm IV.2 as:

\section{Algorithm IV.3: AIMD UTILITY OPTIMIZATION $\left(\boldsymbol{p}_{\boldsymbol{i}}\right)$}

$$
p_{i}(0)=p_{i 0}
$$

\section{repeat}

$$
t=t+1
$$

if $\sum_{i=1}^{n} p_{i}(t)<d(t)$

$$
p_{i}(t+1)=\min \left[p_{i}(t)+\frac{\alpha_{\lambda}}{2 a_{i}}, \bar{p}_{i}(t)\right], \forall i=1, \ldots, n
$$

else

$$
\begin{aligned}
& p_{i}(t+1)= \\
& \max \left[\left(\beta_{\lambda}+b_{i} \frac{\beta_{\lambda}-1}{2 a_{i} p_{i}(t)}\right) \cdot p_{i}(t), \underline{p}_{i}(t)\right], \forall i
\end{aligned}
$$

until end of simulation

\footnotetext{
${ }^{3}$ Because of the discrete nature of AIMD, the proposed approach will provide an optimal solution at the sample times.
}

Note that this algorithm provides the same solution of Algorithm IV.2 with parameters $\alpha_{\lambda}$ and $\beta_{\lambda}$, and it is formally identical to algorithm IV.1, by appropriately changing different parameters for each DER as

$$
\begin{aligned}
\alpha_{i} & =\frac{\alpha_{\lambda}}{2 a_{i}} \\
\beta_{i}(t) & =\beta_{\lambda}+b_{i} \cdot \frac{\beta_{\lambda}-1}{2 a_{i} p_{i}(t)} .
\end{aligned}
$$

Algorithm IV.3 solves the utility optimization problem, while retaining the convenient feature of AIMD that the only required communication is the notification of the balancing event, broadcasted by the EMS to all the DERs. The only required assumption is that each DER knows its own cost function (i.e., parameters $a_{i}, b_{i}$ and $c_{i}$ that associate their own generated power with the financial costs) that are required to tune the personal AIMD parameters $\alpha_{i}$ and $\beta_{i}$.

\section{OPENDSS SIMULATIONS}

\section{A. Simulation Set-Up}

In order to evaluate the performance of our proposed AIMD utility optimization strategy in a more realistic fashion, we tested our algorithms on a revised version of the distribution power system based on the IEEE 37 bus test feeder [38]. This test network incorporates a certain amount of loads, is served by several DERs, and corresponds to the microgrid (or VPP) as illustrated in Fig. 1. Figs. 4 and 5 show the topology of our test network. In our simulations, the base voltage of the High Voltage (HV) network was set to $110 \mathrm{kV}(1.0 \mathrm{pu})$ at the source-end of the external grid. A 2.5 MVA distribution substation was connected to the external grid to bring the voltage level down to $10 \mathrm{kV}$, which is a typical Medium Voltage (MV) level in European power systems. We considered three wind DERs, two PV DERs, and a CHP. Each DER is able to produce power to the MV substation via a connected transformer. To simplify our model, we assumed that the same type of DERs had the same level of generation capacity. However, the real power output from each DER was dependent on the available resource (e.g., wind speed/solar intensity) and the requested power from the load area at each time slot. In addition to this, all the DERs were modeled as constant $\mathrm{P}-\mathrm{Q}$ generators with the same power factors equal to 1.0 to generate pure active power for the loads. It is clearly not realistic to assume that DERs produce pure active power and to model the loads without reactive power consumption. However, we did so as the focus of this paper is on active power generation. In practice, this corresponds to assuming that the reactive power is provided by some ancillary services in the power network (e.g., capacitance tanks, reactive Vehicle-to-Grid (V2G) and Grid-to-Vehicle (G2V) services), or bought from the external grid. Note that the AIMD algorithm could be further extended through a double prioritized algorithm to accomplish reactive power management, as shown in the recent [39] in the context of EV charging, and is not shown here due to page limits.

We also assumed that the wind plants, the PV plants and the CHP had a capacity of $750 \mathrm{~kW}, 200 \mathrm{~kW}$ and $1 \mathrm{MW}$ respectively. Such values typically allow the microgrid to work in island 


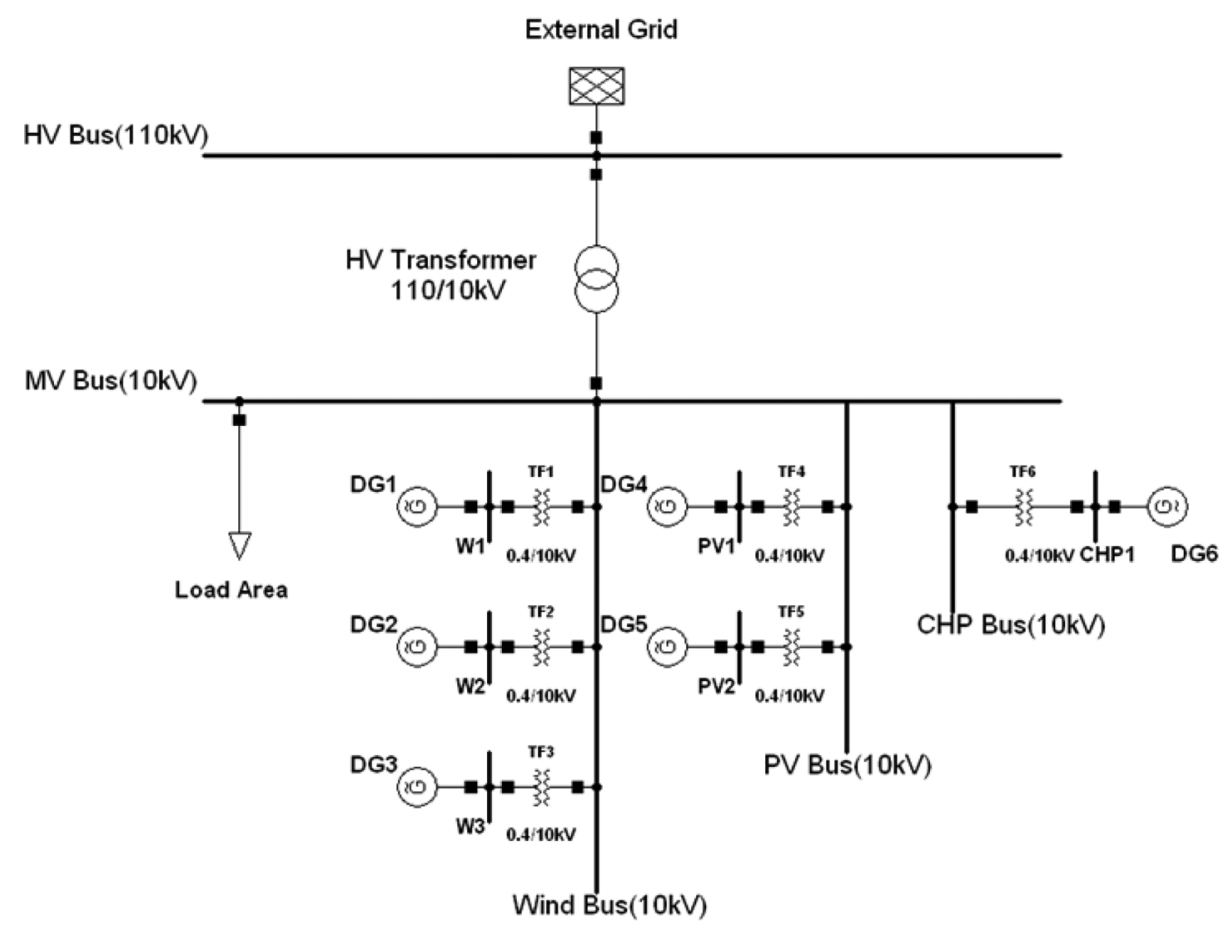

Fig. 4. Schematic topology of the tested network.

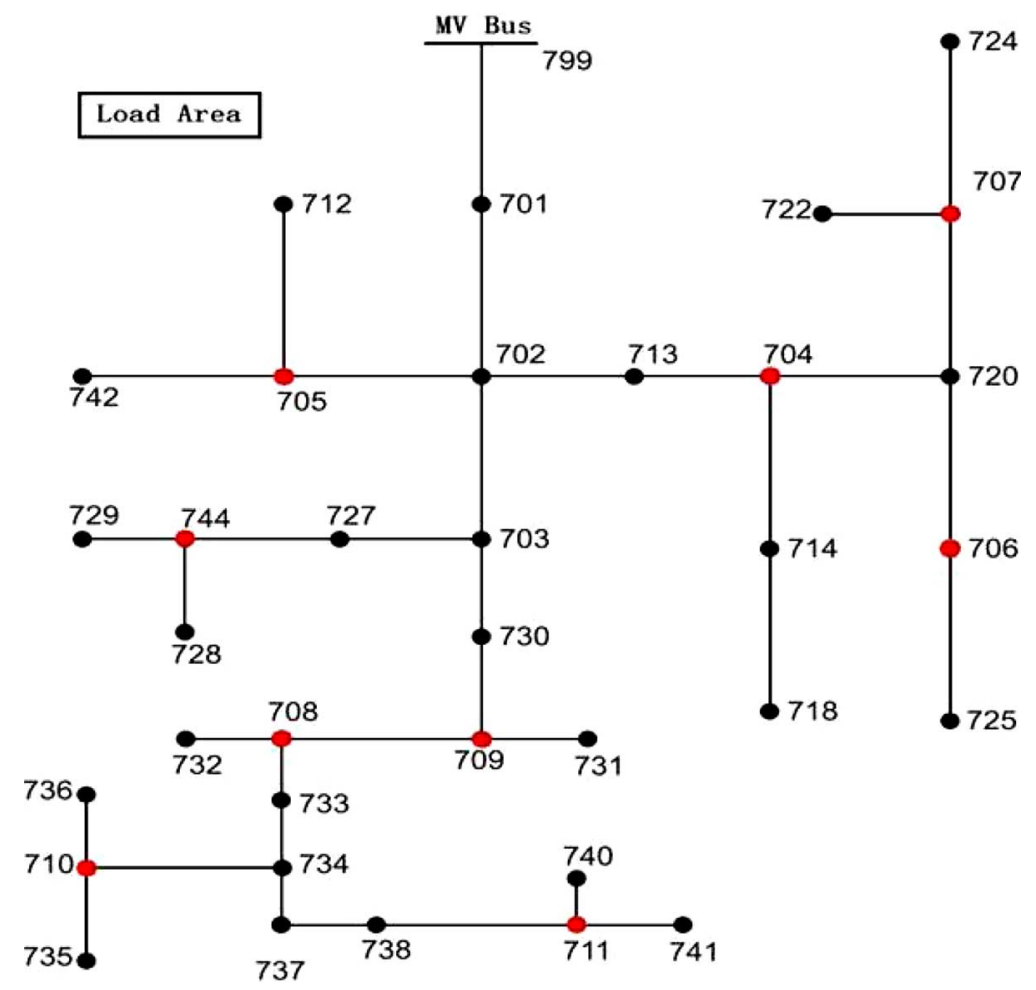

Fig. 5. The IEEE 37 node test feeder was modified as shown in the figure, where the red points were considered without load connections.

mode, i.e., the required power is less than the power provided by the DERs, as assumed in Section III. We assumed that each load had a power factor of 0.95 lagging, and load profiles were randomly chosen for a period of 24 hours, according to [40]. The maximum wind power output for each wind DER was randomly chosen from the real wind turbine data from National Renewable Energy Laboratory (NREL) [41]. The maximum solar power generation profile of each PV was computed according to a quadratic function with non-zero values from 6 am to $6 \mathrm{pm}$, randomly perturbed to simulate cloud disturbances, as in [15]. The parameters of the utility functions were taken from [16] (and converted from euros to US dollars), and [30] and are summarized in Table I. We decided to sample the load profiles and the maximum output of the DERs every 5 minutes, and assumed 

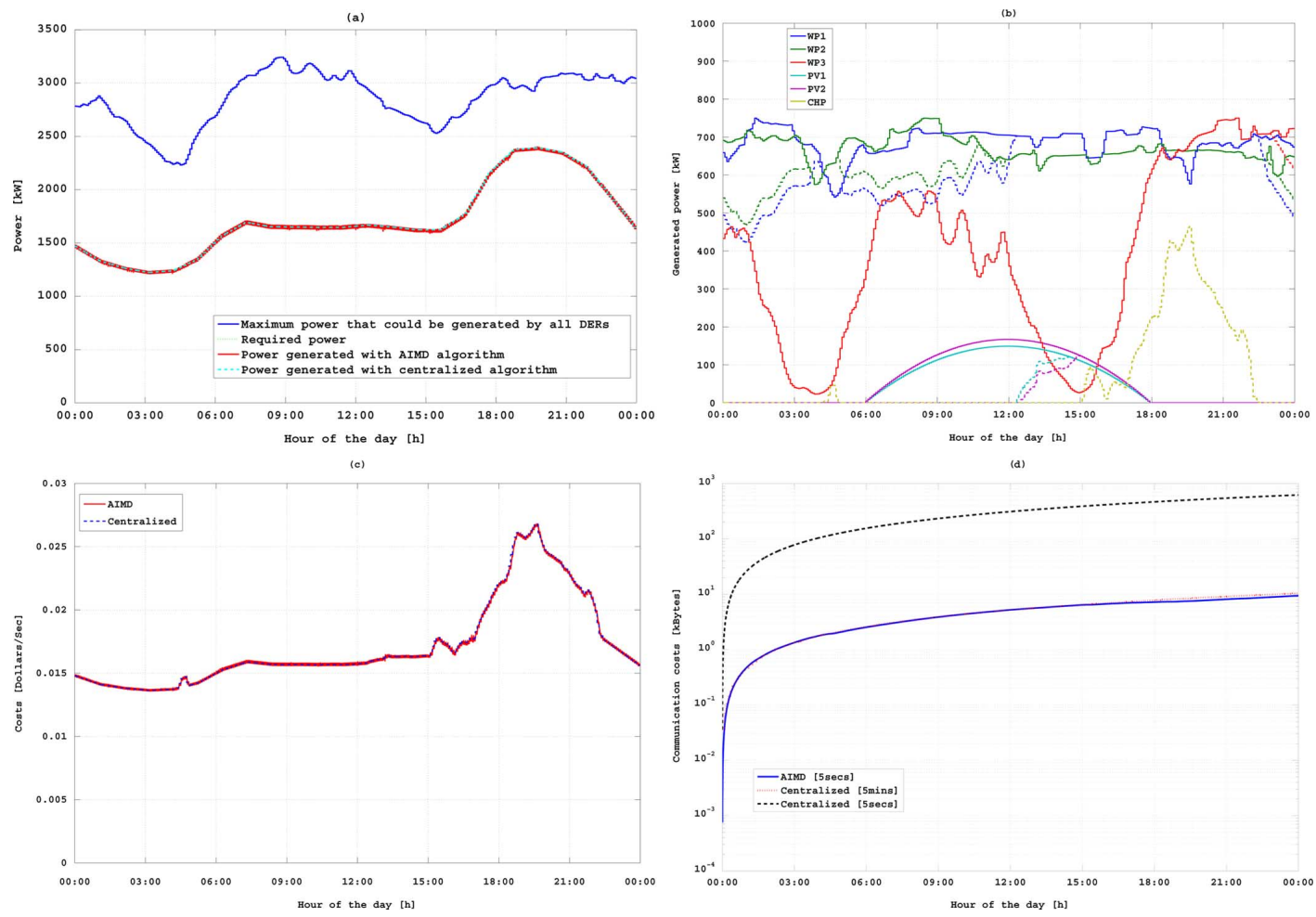

Fig. 6. Figure (a) shows that the distributed AIMD solution manages to balance demand and provided power during the whole day, similarly to the optimal centralized solution. Figure (b) gives the detail of how AIMD shares the energy production. Figure (c) shows that the AIMD manages to achieve the same minimum of the centralized solution. Figure (d) shows compares the communication costs of the two algorithms: with same step size, AIMD clearly outperforms the centralized approach.

TABLE I

PARAMETERS OF THE UTILITY FUNCTIONS

\begin{tabular}{||c||c|c|c||}
\hline Plant & $a_{i}$ & $b_{i}$ & $c_{i}$ \\
\hline Wind Plant 1 & 0.0027 & 17.83 & 4.46 \\
\hline Wind Plant 2 & 0.0028 & 17.54 & 4.45 \\
\hline Wind Plant 3 & 0.0026 & 17.23 & 4.44 \\
\hline Solar PV 1 & 0.0055 & 29.30 & 4.45 \\
\hline Solar PV 2 & 0.0055 & 29.58 & 4.46 \\
\hline CHP & 0.0083 & 75.73 & 5.21 \\
\hline
\end{tabular}

that they would be constant during such a time lapse. The whole scenario was simulated using a customized OpenDSS-Matlab simulation platform [42]. In particular, Matlab was used to generate the dispatch curve for each DER, and a day power system simulation was implemented by OpenDSS to evaluate the state of the network, e.g., line voltage, substation power flow, power losses, for each time slot.

\section{B. Simulation Results}

This section illustrates the simulation results obtained by implementing the proposed AIMD utility optimization algorithm in the network described in the previous section. To better evaluate the performance of the proposed algorithm, we compared the solution with the optimal one obtained in a centralized fashion with a full exchange of information. The centralized solution is computed every 5 minutes, assuming that the EMS is informed of the maximum power that each DER can provide (depending on wind/sun availability) and also by the power required by the users. Then we assumed that the EMS had the ability to solve instantaneously the constrained optimization problem (i.e., considering the power network constraints) and to schedule the optimal power flows to the DERs. As for the AIMD case, we settled with 5 seconds the time step for the increase and decrease steps.

The obtained simulation results are summarized in Fig. 6 . Fig. 6(a) depicts that both the centralized algorithm and the proposed distributed one manage to balance the generated power with that required by the users. We also show the maximum power that could be generated by all the DERs working at full capacity. Fig. 6(b) illustrates how much power was generated by each single DER. As can be noted, the CHP is mainly used to back-up the energy production from the renewable plants, as it is less convenient from the point of view of the cost function being in exam here (i.e., due to its fuel and carbon costs). Fig. 6(c) shows that the value of the utility function is almost the same as would have been obtained by implementing a fully centralized approach. Finally, Fig. 6(d) shows that the communication requirement of the AIMD performed every 5 seconds is similar to that of the centralized solution performed every 5 minutes. However, even in the simple scenario adopted for this comparison, as can be seen from the same figure, the centralized solution can not be used at faster time scales and if we use the same step size for both approaches, then the communication requirement of the centralized approach becomes about 100 times larger than that of AIMD. Clearly, as the operation of smart grids is heading towards real-time fully automated practices, AIMD-like techniques are much more desirable than centralized solution from a communication perspective. 


\section{CONCLUSION}

One of the most interesting and challenging objectives of the upcoming smart grid is the ability to heighten the situational awareness of the grid, and to allow for fast-acting changes in power production and power routing, thus altering the stream of electrical supply and demand on a moment-by-moment basis [1]. Such an ambitious objective poses difficult issues to the energy management system of the power network in terms of communication and control. In this paper we designed AIMD algorithms that manage to minimize a cost function of interest in a microgrid in a distributed manner. The cost function is the total cost of energy production, and this is achieved without any significant communication overhead. In particular, power balancing is achieved without having to communicate real-time power availability from renewable resources (sun/wind), or the power required by the users, but by simply notifying the DERs with a single bit of information every time the provided power equals the required power. The proposed algorithms perform in practice as well as a centralized full-communication algorithm.

Our approach was tested on a simple scenario with a few DERs and a total load of the order of a few MW. The work can be extended in a number of directions: reference [39] shows in a different context, how AIMD algorithms can be modified to further include reactive power management; reference [43] shows how thermal energy requirements can be further included in the microgrid operation; also, it would be interested to check how the presence of several DERs connected to the same line feeder will affect the performance of the proposed method. Accordingly, current work of the authors is continuing along these lines, and will consider a more realistic scenario to further validate the proposed power generation strategies. Also, the suggested strategies will be tested in a small testbed. The oscillations caused by the AIMD method are similar to those that are typically caused by fluctuating renewable sources, thus existing methods used to alleviate the impact of renewables on the grid (e.g., PI smoothing of the generated output, batteries for primary frequency control and voltage control) can be used as well. However, only testing in the real test-bed will definitely validate the proposed method.

\section{ACKNOWLEDGMENT}

The authors would like to thank the (anonymous) reviewers whose remarks contributed to improve the quality and the clarity of the final version of this article.

\section{REFERENCES}

[1] P. Fox-Penner, "Smart Power: Climate Change, the Smart Grid, and the Future of Electric Utilities". Washington, DC, USA, 2010.

[2] "New ERA for Electricity in Europe, Distributed Generation: Key Issues, Challenges and Proposed Solutions," European commission-community research. Brussels, Belgium, 2003.

[3] US Department of Energy, "The Smart Grid: an Introduction," Prepared by Litos Strategic Communication, 2008.

[4] S. Silberman, The Energy Web The Wired, available online at URL [Online]. Available: http://www.wired.com/wired/archive/9.07/juice. html, 2001

[5] W. Su, H. Rahimi-Eichi, W. Zeng, and M.-Y. Chow, "A survey on the electrification of transportation in a smart grid environment," IEEE Trans. Ind. Inf., vol. 8, 1, no. 1, pp. 1-10, 2012.

[6] K. El Bakari and W. L. Kling, "Virtual power plants: An answer to increasing distributed generation," in Proc. Innovative Smart Grid Technologies Conf. (Europe), Gothenburg, Sweden, 2010.
[7] P. Lombardi, M. Powalko, and K. Rudion, "Optimal operation of a virtual power plant," in IEEE Power Energy Soc. General Meeting, Calgary, AB, Canada, 2009.

[8] E. Ancillotti, R. Bruno, and M. Conti, "Smoothing peak demands through aggregate control of background electrical loads," in Proc. IEEE PES Innovative Smart Grid Technologies (ISGT), Washington, 2014.

[9] Y. A.-R. I. Mohamed and A. A. Radwan, "Hierarchical control system for robust microgrid operation and seamless mode transfer in active distribution systems," IEEE Trans. Smart Grid, vol. 2, no. 2, pp. 352-362, Jun. 2011.

[10] D. Chiu and R. Jain, "Analysis of the increase/decrease algorithm for congestion avoidance in computer networks," J. Computer Netw., vol. 17, pp. 1-14, 1989.

[11] P. Palensky and D. Dietrich, "Demand side management: Demand response, intelligent energy systems, and smart loads," IEEE Trans. Ind. Inf., vol. 7, no. 3, pp. 381-388, Aug. 2011.

[12] A. Molderink, V. Bakker, M. G. C. Bosman, J. L. Hurink, and G. J. M. Smit, "Management and control of domestic smart grid technology," IEEE Trans. Smart Grid, vol. 1, no. 2, pp. 109-119, 2010.

[13] A.-H. Mohsenian-Rad and A. Leon-Garcia, "Optimal residential load control with price prediction in real-time electricity pricing environments," IEEE Trans. Smart Grid, vol. 1, no. 2, pp. 120-133, Sep. 2010.

[14] G. K. Venayagamoorthy and P. Mitra, "SmartPark shock absorbers for wind farms," IEEE Trans. Energy Conservation, vol. 26, no. 3, pp. 990-992, 2011.

[15] D. Aloini, E. Crisostomi, M. Raugi, and R. Rizzo, "Optimal power scheduling in a virtual power plant," in Proc. Innovative Smart Grid Technologies (ISGT), Manchester, U.K., 2011.

[16] A. Parisio and L. Glielmo, "A mixed integer linear formulation for microgrid economic scheduling," in Proc. IEEE SmartGridComm, Brussels, Belgium, 2011.

[17] A. Parisio and L. Glielmo, "Stochastic model predictive control for economic/environmental operation management of microgrids," in Proc. IEEE Eur. Control Conf. (ECC), Zürich, Switzerland, 2013.

[18] M. Giuntoli and D. Poli, "Optimized thermal and electrical scheduling of a large scale virtual power plant in the presence of energy storages," IEEE Trans. Smart Grid, vol. 4, no. 2, pp. 942-955, Jun. 2013.

[19] C. Ramsay and M. Aunedi, Characterisation of LSVPPs Fenix project, Del. D1.4.1., available online at url: [Online]. Available: http:// fenix.iwes.fraunhofer.de/docs/documents/Project_Fenix_2009-0706_D1_4_1_Characterisation_of_LSVPPs

[20] M. Li and P. B. Luh, "A decentralized framework of unit commitment for future power markets," in Proc. IEEE and Energy Society General Meeting (PES), Vancouver, BC, Canada, 2013.

[21] W. Su, J. Wang, and J. Roh, "Stochastic energy scheduling in microgrids with intermittent renewable energy resources," IEEE Trans. Smart Grid, to be published.

[22] S. Stüdli, E. Crisostomi, R. Middleton, and R. Shorten, "AIMD-like algorithms for charging electric and plug-in hybrid vehicles," in Proc. IEEE Int. Electric Vehicle Conf., Greenville, SC, USA, 2012.

[23] S. Stüdli, E. Crisostomi, R. Middleton, and R. Shorten, "A flexible distributed framework for realising electric and plug-in hybrid vehicle charging policies," Int. J. Contr., vol. 85, no. 8, pp. 1130-1145, 2012.

[24] I. Beil and I. Hiskens, "A distributed wireless testbed for plug-in hybrid electric vehicle control algorithms," in Proc. North American Power Symp., Champaign, IL, USA, 2012.

[25] C.-K. Wen, J.-C. Chen, J.-H. Teng, and P. Ting, "Decentralized plug-in electric vehicle charging selection algorithm in power systems," IEEE Trans. Smart Grid, vol. 3, no. 4, pp. 1779-1789, Dec. 2012.

[26] Z. Fan, "Distributed demand response and user adaptation in Smart Grids," in Proc. IFIP/IEEE Int. Symp. Integrated Network Management, Dublin, Ireland, 2011.

[27] A. D. Dominguez-Garcia and C. N. Hadjicostis, "Coordination and control of distributed energy resources for provision of ancillary services," in Proc. IEEE Conf. Smart Grid Commun., Gaithersburg, MD, USA, 2010.

[28] R. Caldon, A. R. Patria, and R. Turri, "Optimal control of a distribution system with a virtual power plant," in Proc. Bulk Power System Dynamics Control Conf., Cortina d'Ampezzo, Italy, 2004.

[29] Y. S. Kim, I. K. Eom, K. Y. Lee, and J. H. Park, "Economic load dispatch for piecewise quadratic cost function using Hopfield neural network," IEEE Trans. Power Syst., vol. 8, no. 3, pp. 1030-1038, Aug. 1993.

[30] "International energy agency and organisation for economic co-operation and development-Nuclear energy agency," Projected Costs of Generating Electricity 2010 Edition, 2010. 
[31] R. Srikant, "The mathematics of Internet Congestion Control," in Systems \& Control: Foundations \& Applications. New York, NY, USA: Birkhäuser, 2003.

[32] M. Corless and R. Shorten, "An ergodic AIMD algorithm with application to high-speed networks," Int. J. Contr., vol. 85, no. 6, pp. 746-764, 2012.

[33] R. Shorten, D. Leith, J. Foy, and R. Kilduff, "Analysis and design of AIMD congestion control algorithms in communication networks," Automatica, vol. 41, no. 4, pp. 725-730, 2005.

[34] R. Shorten, F. Wirth, and D. Leith, "A positive systems model of TCPlike congestion control: Asymptotic results," IEEE/ACM Trans. Netw., vol. 14 , no. 3 , pp. 616-629, Jun. 2006

[35] M. Corless and R. Shorten, "Analysis of a general nonlinear increase-decrease resource allocation algorithm with application to network utility maximization," submitted for publication.

[36] K. Kar, S. Sarkar, and L. Tassiulas, "A simple rate control algorithm for max total user utility," in Proc. IEEE INFOCOM, 2001, vol. 1.

[37] R. Stanojevic and R. Shorten, "Fully decentralised emulation of besteffort and processor sharing queues," in Proc. ACM Sigmetrics, 2008, pp. 383-394.

[38] IEEE distribution system analysis subcommittee, IEEE Trans. Power Syst., vol. 6, no. 3, pp. 975-985, Aug. 1991.

[39] S. Stüdli, E. Crisostomi, R. Middleton, and R. Shorten, "Optimal realtime distributed V2G and G2V management of electric vehicles," Int. J. Contr., vol. 87, no. 6, pp. 1153-1162, 2014.

[40] South California Edison [Online]. Available: http://www.sce.com/ 005 regul info/eca/DOMSM11.DLP

[41] National Sustainable Energy Laboratory (NREL) [Online]. Available: https://pfs.nrel.gov/main.html

[42] OpenDSS Program [Online]. Available: http://sourceforge.net/ projects/electricdss

[43] M. Liu, E. Crisostomi, M. Raugi, and R. Shorten, "Optimal distributed power generation for thermal and electrical scheduling in a microgrid," in Proc. Innovative Smart Grid Technologies (ISGT), Copenhagen, 2013.

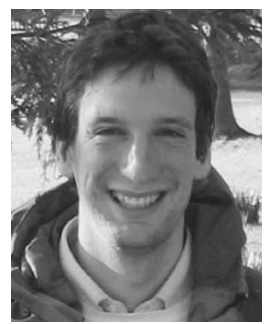

Emanuele Crisostomi received the B.S. degree in computer science engineering, the M.S. degree in automatic control, and the Ph.D. degree in automatics, robotics, and bioengineering, from the University of Pisa, Italy, in 2002, 2005, and 2009, respectively.

$\mathrm{He}$ is currently an Assistant Professor of electrical engineering with the Department of Energy, Systems, Territory and Constructions Engineering, University of Pisa. His research interests include control and optimization of large-scale systems, with applications to smart grids and green mobility networks.

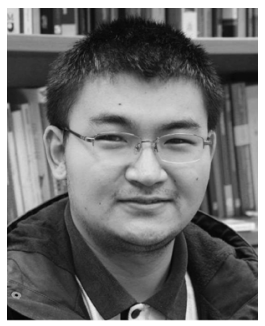

Mingming Liu received the B.E. degree in electronics engineering (first class honors) from National University of Ireland, Maynooth (NUI Maynooth) in 2011. He is currently working towards a Ph.D. degree in applied mathematics under supervision of Prof. Robert Shorten at the Hamilton Institute, NUI Maynooth.

His current research interests are smart grid analysis, modelling and optimisation, EV charging strategies and distributed control techniques.

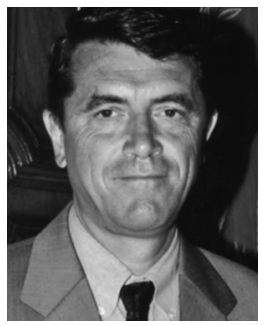

Marco Raugi received the Ph.D. degree in electrical engineering from the University of Pisa, Pisa, Italy, in 1990.

Currently, he is a Full Professor of Electrical Engineering with the Department of Energy, Systems, Territory and Constructions Engineering, University of Pisa. He is the author of many papers in international journals and conference proceedings. His research interests include numerical electromagnetics, with main applications in nondestructive testing, electromagnetic compatibility, communications, and computational intelligence.

Prof. Raugi was the General Chairman of the international conferences Progress in Electromagnetic Research Symposium in 2004 and IEEE International Symposium on Power Line Communications in 2007. He was the recipient of the IEEE Industry Application Society 2002 Melcher Prize Paper Award.

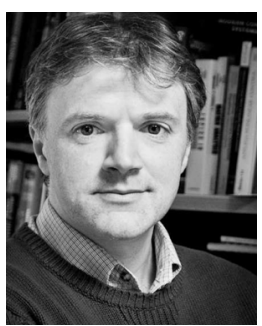

Robert Shorten received the B.E. degree (first-class honors) from the University College Dublin (UCD) in 1990 in electronic engineering. He received the Ph.D. degree in 1996, also from UCD, while based in at Daimler-Benz Research in Berlin, Germany.

From 1993 to 1996, he was the holder of a Marie Curie Fellowship at Daimler-Benz Research to conduct research in the area of smart gearbox systems. Following a brief spell at the Center for Systems Science, Yale University, working with Professor K. S. Narendra, he returned to Ireland as the holder of a European Presidency Fellowship in 1997. He is a co-founder of the Hamilton Institute at NUI Maynooth, where he was a full Professor until March 2013. He was also a Visiting Professor at TU Berlin from 2011-2012. He is currently a Senior Research Manager at IBM Research Ireland. His research spans a number of areas. He has been active in computer networking, automotive research, collaborative mobility (including smart transportation and electric vehicles), as well basic control theory and linear algebra. His main field of theoretical research has been the study of hybrid dynamical systems. 